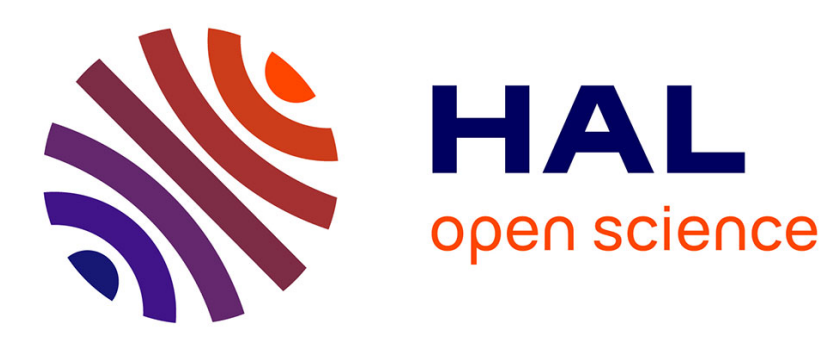

\title{
Mécanisme d'excitation des molécules polyatomiques par l'azote actif dans des matrices argon-azote
}

\author{
Emmanuel Faure, F. Valadier, J. Janin
}

\section{To cite this version:}

Emmanuel Faure, F. Valadier, J. Janin. Mécanisme d'excitation des molécules polyatomiques par l'azote actif dans des matrices argon-azote. Journal de Physique, 1975, 36 (2), pp.117-124. 10.1051/jphys:01975003602011700 . jpa-00208235

\section{HAL Id: jpa-00208235 https://hal.science/jpa-00208235}

Submitted on 1 Jan 1975

HAL is a multi-disciplinary open access archive for the deposit and dissemination of scientific research documents, whether they are published or not. The documents may come from teaching and research institutions in France or abroad, or from public or private research centers.
L'archive ouverte pluridisciplinaire HAL, est destinée au dépôt et à la diffusion de documents scientifiques de niveau recherche, publiés ou non, émanant des établissements d'enseignement et de recherche français ou étrangers, des laboratoires publics ou privés. 


\title{
Classification \\ Physics Abstracts 5.447 \\ MÉCANISME D'EXCITATION DES MOLÉCULES POLYATOMIQUES PAR L'AZOTE ACTIF DANS DES MATRICES ARGON-AZOTE
}

\author{
E. FAURE, F. VALADIER et J. JANIN \\ Laboratoire de Spectroscopie et de Luminescence $\left(^{*}\right)$ \\ Université Claude-Bernard, Lyon I, 43, boulevard du 11-novembre-1918, 69621 Villeurbanne, France
}

(Reçu le 19 février 1974, révisé le 15 juillet 1974)

\begin{abstract}
Résumé. - On a cherché à obtenir des informations sur le mécanisme d'excitation des molécules polyatomiques par l'azote actif dans des matrices d'azote ou d'azote et d'argon, en étudiant la variation des intensités du système de Vegard-Kaplan de l'azote et des bandes de plusieurs produits carbonés dans des matrices argon-azote, en fonction de la concentration du composé et de la température. Il n'est pas possible de conclure avec certitude, étant donné la complexité des phénomènes. Toutefois, l'hypothèse d'un transfert d'énergie par échange électronique entre les molécules $\mathrm{N}_{2}\left(\mathrm{~A}^{3} \Sigma_{\mathrm{u}}^{+}\right)$et les molécules polyatomiques rend assez bien compte des résultats expérimentaux. L'influence de la diffusion des molécules d'azote métastables a été également examinée.
\end{abstract}

\begin{abstract}
In order to obtain information about the excitation mechanism of polyatomic molecules by active nitrogen in $N_{2}$ and $A-N_{2}$ matrices, we have studied intensity variations in the Vegard-Kaplan system and in the bands of a number of carbon compounds in argon-nitrogen matrices, as functions of compound concentration and temperature. Considering the complexity of the phenomena, it is not possible to give an absolute conclusion about this process, but the hypothesis of an energy transfer by exchange interaction between $\mathrm{N}_{2}\left(\mathrm{~A}^{3} \Sigma_{\mathrm{u}}^{+}\right)$molecules and polyatomic molecules appears to explain reasonably well the experimental results. The effect of the diffusion of metastable nitrogen molecules has also been investigated.
\end{abstract}

On a décrit dans des publications antérieures les spectres émis par diverses molécules polyatomiques (benzène et ses dérivés, acétonitrile, propionitrile, cyanogène, acide cyanhydrique, ...) excitées par l'azote actif dans des matrices d'azote ou d'argon à $20 \mathrm{~K}$ [1].

Il s'agissait, dans tous les cas où les états triplets inférieurs des molécules étudiées étaient connus, des transitions $\mathrm{T} \rightarrow \mathrm{S}$ entre cet état triplet et l'état singulet fondamental de la molécule. On a donc supposé que l'excitation se produisait suivant le schéma général :

$$
\mathrm{N}_{2}\left(\mathrm{~A}^{3} \Sigma_{\mathrm{u}}^{+}\right)+\mathrm{X}(\mathrm{S}) \rightarrow \mathrm{N}_{2}\left(\mathrm{X}^{1} \Sigma_{\mathrm{g}}^{+}\right)+\mathrm{X}(\mathrm{T})
$$

Un tel mécanisme dans lequel le spin total des molécules en interaction est conservé, doit, en effet, être très probable. Cette hypothèse $a$, d'ailleurs, reçu une première confirmation par les expériences de Meyer, Stedman et Setser [2] sur l'excitation de la molécule $\mathrm{C}_{2} \mathrm{~N}_{2}$ par l'azote actif en phase gazeuse.

Il convenait néanmoins d'étudier plus en détail le mécanisme d'excitation en phase condensée, qui intervient dans le procédé mis en œuvre dans notre

(*) Equipe de Recherche associée au C.N.R.S. $n^{\circ} 302$. laboratoire, afin de préciser si possible la nature du transfert d'énergie entre l'azote excité et les molécules étrangères. C'est l'objet du travail décrit ici.

1. Dispositif expérimental. - Un courant d'azote pur ou d'un mélange contenant de l'argon et quelques pour-cent d'azote est soumis en A (Fig. 1) à un champ de très haute fréquence produit soit par un générateur d'ondes centimétriques fonctionnant en impulsions (longueur d'onde $3,2 \mathrm{~cm}$, durée des impulsions $1 \mu \mathrm{s}$, fréquence de répétition $400 \mathrm{~Hz}$ ), soit par un générateur continu de $2500 \mathrm{MHz}$. On injecte en $\mathrm{B}$ dans le gaz excité une petite quantité du composé polyatomique et le mélange est condensé sur le doigt froid D d'un cryostat en verre pyrex dont le schéma est représenté sur la figure 1 . Le réservoir central $R$ contenant l'hélium ou l'hydrogène liquide est entouré d'une gaine $\mathrm{G}$ d'azote liquide jusqu'à sa base. Il est prolongé par le doigt parallélépipédique $\mathrm{D}$ sur lequel s'effectue la condensation. L'ensemble se trouve à l'intérieur d'une enceinte $\mathrm{E}$ reliée au groupe de pompage. Cette enceinte porte au niveau du doigt trois tubulures T fermées par des glaces de silice Suprasil S. Les deux premières, disposées à $180^{\circ}$ l'une de l'autre, 


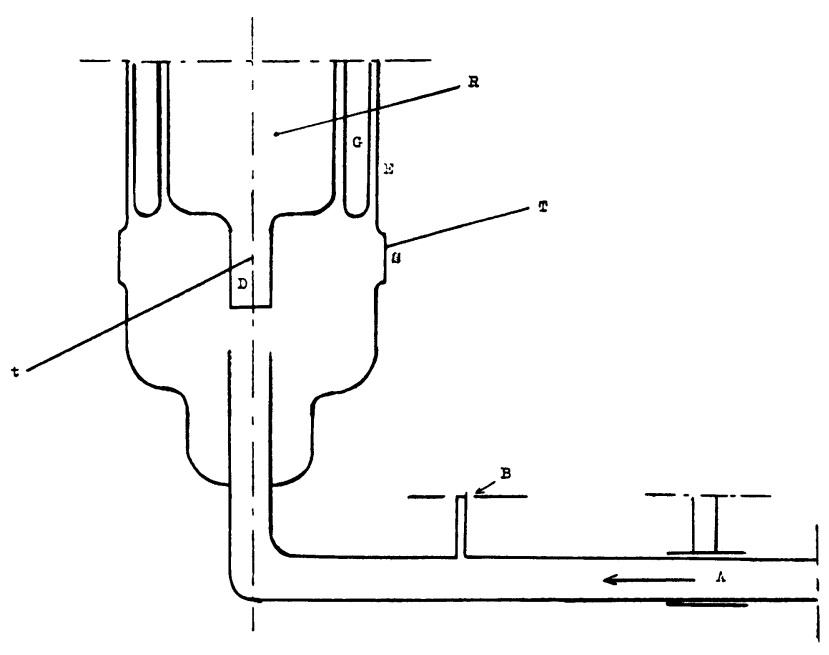

FIG. 1. - Schéma du dispositif.

sont destinées à l'observation de la luminescence du dépôt solide et la troisième, dont l'axe est orienté à $30^{\circ}$ de celui des deux autres, est utilisée pour irradier le dépôt par une lampe à vapeur de mercure lorsqu'on veut exciter le spectre de fluorescence du composé étudié.

On a pu ainsi enregistrer le spectre du rayonnement émis au moyen d'un spectrographe Hilger Medium et étudier simultanément les variations d'intensité d'une radiation à l'aide d'un monochromateur Jobin et Yvon HRS 2 associé à un photomultiplicateur EMI 9526 B.

Un couple thermoélectrique or-fer/chromel était fixé en $t$ sur le doigt froid du cryostat. Il indiquait une température voisine de $10 \mathrm{~K}$ lorsqu'on opérait en régime dynamique avec l'hélium liquide et de $25 \mathrm{~K}$ quand, dans les mêmes conditions, on refroidissait par l'hydrogène liquide.

2. Conditions d'observation des spectres et mesures effectuées. - La luminescence de l'azote condensé à basse température après excitation dans une décharge électrique a déjà fait l'objet de nombreux travaux [3]. On se bornera ici à comparer les résultats des observations effectuées en l'absence et en présence du composé polyatomique.

Lorsqu'on opère avec l'azote seul, la luminescence est faible au-dessous de $10 \mathrm{~K}$. Il apparaît de façon intermittente des éclairs qui traversent le dépôt solide et qui sont suivis d'une forte phosphorescence de couleur verte. Le spectre correspondant est constitué essentiellement des raies de l'azote atomique et de celles de l'oxygène $\mathrm{OI}\left({ }^{1} \mathrm{~S} \rightarrow{ }^{1} \mathrm{D}\right)[3]$. Au voisinage de $20 \mathrm{~K}$, en revanche, la luminescence qui est jaune verdâtre, est intense et continue. La composition spectrale du rayonnement est différente. On constate notamment un renforcement considérable de l'émission de l'oxygène et la présence avec une faible intensité des bandes de Vegard-Kaplan de $\mathrm{N}_{2}$

$$
\left(\mathrm{A}^{3} \Sigma_{\mathrm{u}}^{+} \rightarrow \mathrm{X}^{1} \Sigma_{\mathrm{g}}^{+}\right) .
$$

Si l'on remplace l'azote par un mélange d'argon et d'azote (contenant 1 à $10 \%$ de ce dernier gaz), l'émission est encore très faible à la température de l'hélium liquide, en dehors des éclairs qui, dans ces conditions, sont très rares. Mais, vers $20 \mathrm{~K}$, il apparaît une luminescence bleue intense. Son spectre est surtout constitué des bandes de Vegard-Kaplan, le groupe de raies $\mathrm{N}\left({ }^{2} \mathrm{D} \rightarrow{ }^{4} \mathrm{~S}\right)$ n'ayant alors qu'une faible intensité.

Ces mêmes émissions sont observées lorsque, ayant condensé l'azote ou le mélange argon-azote sur la paroi refroidie par l'hélium liquide, on supprime l'arrivée de gaz et on laisse lentement se réchauffer le dépôt solide.

Quand on injecte dans le courant d'azote excité le composé polyatomique à une concentration de l'ordre de $1 \%$, la luminescence verte est remplacée par une émission bleu-violette. Les conditions d'observation de cette émission sont identiques à celles de la luminescence fournie par les gaz purs. Très faible au-dessous de $10 \mathrm{~K}$ en dehors des éclairs, elle présente une forte intensité à $20 \mathrm{~K}$.

Pour étudier les transferts d'énergie entre les molécules métastables $\mathrm{N}_{2}\left(\mathrm{~A}^{3} \Sigma_{\mathrm{u}}^{+}\right)$et les molécules carbonées $\mathrm{X}$, on a comparé les variations respectives des intensités de la bande $\lambda=2950 \AA(0-7)$ du système $\mathrm{A}^{3} \Sigma_{\mathrm{u}}^{+} \rightarrow \mathrm{X}^{1} \Sigma_{\mathrm{g}}^{+}$et celles de l'une des bandes $\mathrm{du}$ systèmè $\mathrm{T} \rightarrow \mathrm{S}$ du composé $\mathrm{X}\left(\mathrm{C}_{6} \mathrm{H}_{6}\right.$ ou $\left.\mathrm{C}_{2} \mathrm{~N}_{2}\right)$, lorsqu'on fait varier la concentration de ce dernier, en régime stationnaire à $25 \mathrm{~K}$, d'une part, et lorsque la température s'élève pendant la période de réchauffement, pour une teneur donnée du composé polyatomique, d'autre part.

Les mesures ont porté sur des mélanges argonazote de concentration en azote $6 \%$. Dans une matrice d'azote pur, en effet, les transferts d'énergie par exciton entre les molécules d'azote jouent un rôle très important, si bien que l'énergie d'excitation des molécules $\mathrm{N}_{2}\left(\mathrm{~A}^{3} \Sigma_{\mathrm{u}}^{+}\right)$est finalement transmise aux atomes $\mathrm{N}$ et $\mathrm{O}$ présents en petite quantité dans la matrice [4] et les bandes de Vegard-Kaplan ont une faible intensité. Ce n'est pas le cas dans une matrice argon-azote, puisque la raie interdite de l'oxygène est invisible et que la raie $\mathrm{N}\left({ }^{2} \mathrm{D} \rightarrow{ }^{4} \mathrm{~S}\right)$ est peu intense. Aussi peut-on, en première approximation, négliger l'influence des transferts entre molécules métastables et atomes d'azote et considérer que la désexcitation des molécules $\mathrm{N}_{2}\left(\mathrm{~A}^{3} \Sigma_{\mathrm{u}}^{+}\right)$est surtout radiative. On admettra donc qu'en présence du composé polyatomique $X$, se produit essentiellement une compétition entre l'émission spontanée des molécules métastables et le transfert sans rayonnement de leur énergie aux molécules $\mathrm{X}$.

3. Résultats des mesures. - 3.1 INFLUENCE DE LA CONCENTRATION. - Les courbes de la figure 2 représentent les variations à $25 \mathrm{~K}$ des intensités des bandes $\lambda=2950 \AA$ de l'azote et

$$
\lambda=3519 \AA \quad\left(v_{9}, v=1\right)
$$




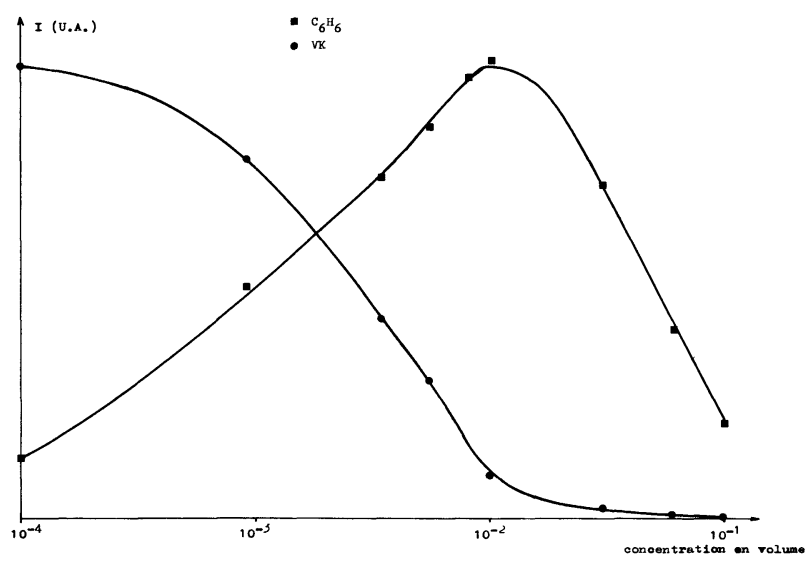

FIG. 2. - Variation avec la concentration du benzène des intensités des bandes (0-7) de Vegard-Kaplan et $\left(v_{9}, v=1\right)$ de $\mathrm{C}_{6} \mathbf{H}_{6}$.

du système $\mathrm{T} \rightarrow \mathrm{S}$ du benzène, quand on fait croître la concentration de ce composé dans le mélange. On constate, comme on devait s'y attendre d'après le schéma (1), une diminution progressive de l'intensité de la bande de l'azote qui s'accompagne d'un accroissement de celle de $\mathrm{C}_{6} \mathrm{H}_{6}$. Quand la concentration en volume dépasse $10^{-2}$ environ, il se produit une extinction progressive de la luminescence du benzène. Les phénomènes observés dans le cas du cyanogène sont similaires (Fig. 3).

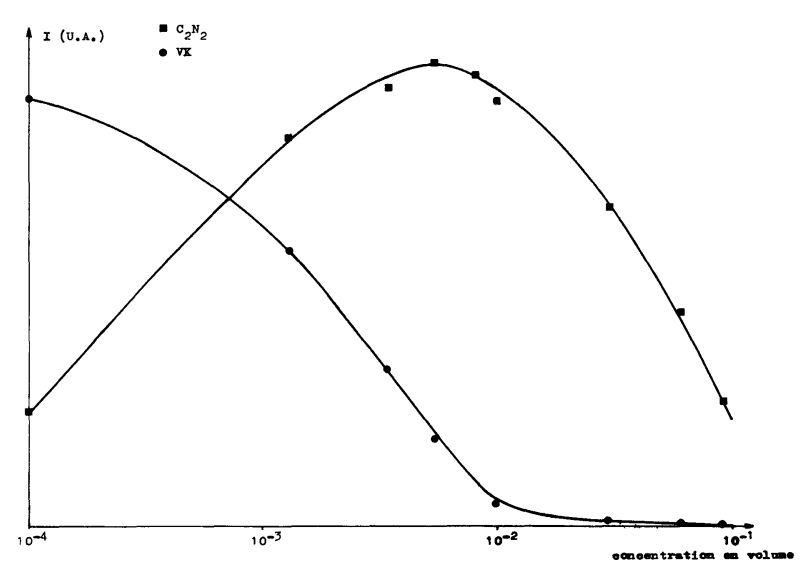

Fig. 3. - Variation avec la concentration du cyanogène des intensités des bandes (0-7) de Vegard-Kaplan et $\left(v_{1}, v=2\right)$ de $\mathrm{C}_{2} \mathrm{~N}_{2}$.

\subsection{EFFET D'UNE ÉLÉVATION DE TEMPÉRATURE SUR} L'INTENSITÉ DES BANDES. - On a représenté sur les figures 4 à 6 les variations des intensités des bandes étudiées lorsque, ayant réalisé le dépôt solide à la température de l'hélium liquide et supprimé le courant gazeux, on laisse l'hélium s'évaporer et le cryostat s'échauffer naturellement. On constate que l'émission des bandes de Vegard-Kaplan débute vers $10 \mathrm{~K}$ en l'absence comme en présence du produit carboné et que dans ce dernier cas, leur intensité diminue beaucoup au-dessus de $25 \mathrm{~K}$, tandis qu'à partir de cette température le spectre du composé carboné devient très intense.

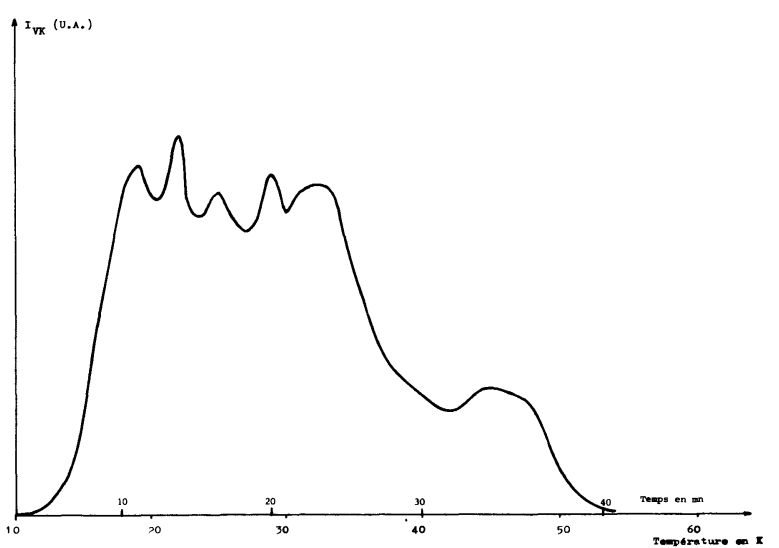

Fig. 4. - Variation avec la température de l'intensité de la bande (0-7) de Vegard-Kaplan dans un mélange argon-azote $(6 \%)$ (Les temps de réchauffement sont indiqués en abscisses.)

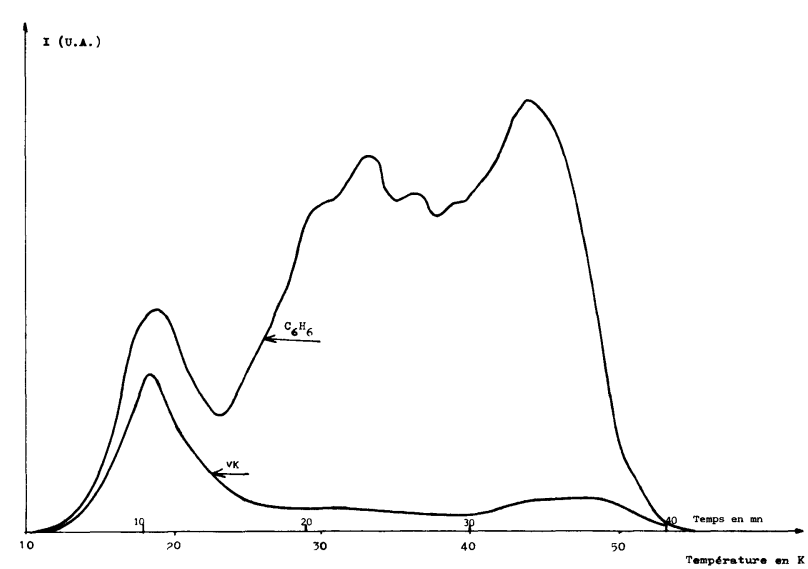

Fig. 5. - Variation avec la température des intensités des bandes $(0-7)$ de Vegard-Kaplan et $\left(v_{9}, v=1\right)$ de $\mathrm{C}_{6} \mathrm{H}_{6}$ dans un mélange argon-azote $(6 \%)$, benzène $(1 \%)$. (Les temps de réchauffement sont indiqués en abscisses.)

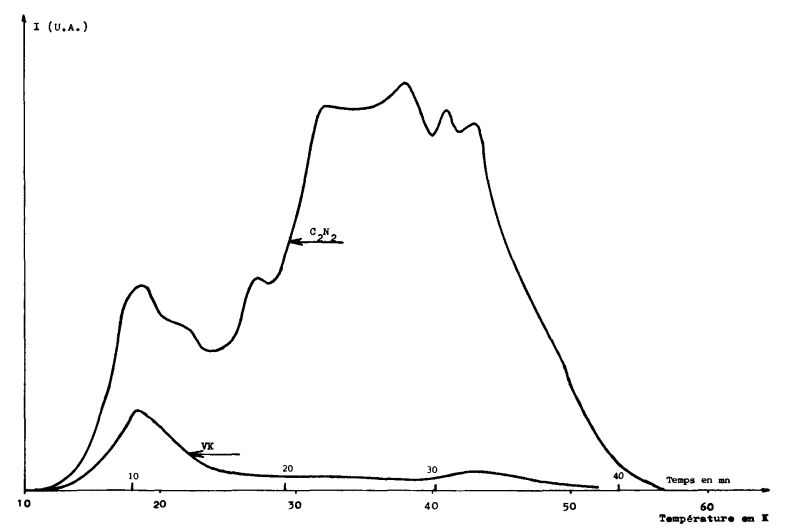

Fig. 6. - Variation avec la température des intensités des bandes (0-7) de Vegard-Kaplan et $\left(v_{1}, v=2\right)$ de $\mathrm{C}_{2} \mathrm{~N}_{2}$ dans un mélange argon-azote $(6 \%)$, cyanogène $(1 \%)$. (Les temps de réchauffement sont indiqués en abscisses.)

La courbe tracée sur la figure 4 donne l'intensité moyenne de la bande $\lambda=2950 \AA$ de l'azote, alors que les enregistrements obtenus montrent qu'en réalité, la variation de l'émission n'est pas continue, mais qu'elle présente un grand nombre de maximums 
répartis de façon aléatoire, ce qui semble indiquer que la température de la matrice n'est pas uniforme (Fig. 7). Cet effet est beaucoup moins marqué pour la bande $\lambda=3519 \AA$ du benzène (Fig. 8), par suite vraisemblablement de la longueur de la durée de vie des molécules $\mathrm{C}_{6} \mathrm{H}_{6}(\mathrm{~T})$ très supérieure à celle des molécules $\mathrm{N}_{2}\left(\mathrm{~A}^{3} \Sigma_{\mathrm{u}}^{+}\right)$.

La variation avec la température de l'intensité de la bande $\lambda=3500 \AA\left(v_{1}, v=2\right)$ de $\mathrm{C}_{2} \mathrm{~N}_{2}$ diffère assez peu de celle des bandes du benzène, comme on le voit sur la figure 6.

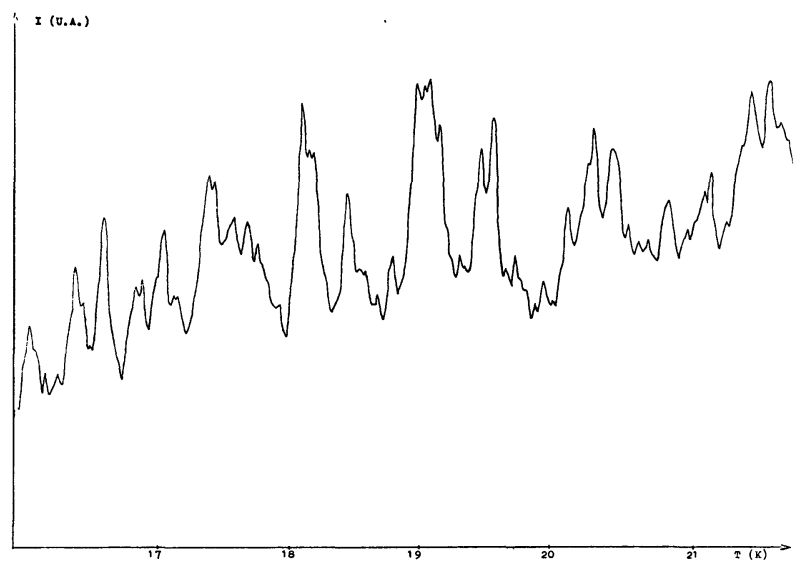

Fig. 7. - Enregistrement de la variation avec la température de la bande (0-7) de Vegard-Kaplan dans un mélange argon-azote (6\%).

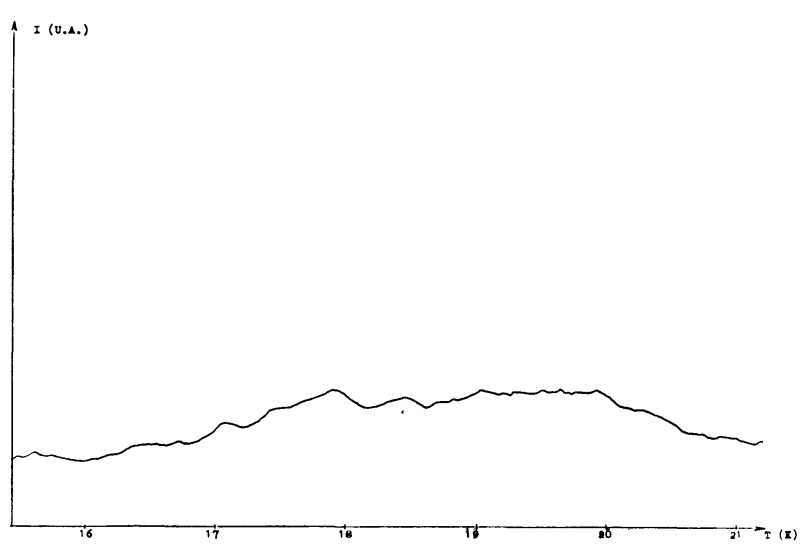

FIG. 8. - Enregistrement de la variation avec la température de la bande $\left(v_{9}, v=1\right)$ de $\mathrm{C}_{6} \mathrm{H}_{6}$ dans un mélange argon-azote $(6 \%)$, benzène $(1 \%)$.

4. Discussion des résultats. -4.1 FORMATION DES MOlÉCules $\mathrm{N}_{2}\left(\mathrm{~A}^{3} \Sigma_{\mathrm{u}}^{+}\right)$. - Comme la durée de vie des molécules $\mathrm{N}_{2}\left(\mathrm{~A}^{3} \Sigma_{\mathrm{u}}^{+}\right)$est $0,35 \mathrm{~s}$ à $4,2 \mathrm{~K}$ dans une matrice d'argon contenant $1 \%$ d'azote [5], on conclut des résultats précédents que les molécules qui jouent un rôle prépondérant dans le mécanisme d'excitation ne peuvent être formées en phase gazeuse. Elles doivent donc provenir de la recombinaison des atomes $\mathrm{N}\left({ }^{4} \mathrm{~S}\right)$ présents dans l'azote actif et piégés dans la matrice [6]. Ces atomes pouvant migrer dans le réseau à partir de $10 \mathrm{~K}$, on peut ainsi expliquer. la forte émission du système $\mathrm{A}^{3} \Sigma_{\mathrm{u}}^{+} \rightarrow \mathrm{X}^{1} \Sigma_{\mathrm{g}}^{+}$au-dessus de cette température.
Quand on opère en régime dynamique à $25 \mathrm{~K}$, la recombinaison se produit principalement dans la couche superficielle du dépôt, car la quantité d'atomes piégés au sein de la matrice à cette température est faible, ce que montrent les deux faits suivants : d'une part, la luminescence cesse très rapidement après la suppression du courant gazeux, et d'autre part, un réchauffement à partir de $25 \mathrm{~K}$ n'est accompagné d'aucune émission. C'est dans cette couche superficielle qui présente certainement de nombreux défauts, que sont formées les molécules excitées et qu'ont lieu les transferts d'énergie, dans les conditions où ont été effectuées les mesures d'intensité des bandes.

4.2 MÉCANISMES DE TRANSFERT D'ÉNERGIE ENTRE MOLÉCULES $\mathrm{N}_{2}\left(\mathrm{~A}^{3} \Sigma_{\mathrm{u}}^{+}\right)$ET MOLÉCULES POLYATOMIQUES. - 4.2.1 Transfert d'énergie par interaction dipolaire. - On sait que dans le cas d'un transfert d'énergie par interaction dipolaire entre un donneur et un accepteur (ici les molécules $\mathrm{N}_{2}\left(\mathrm{~A}^{3} \Sigma_{\mathrm{u}}^{+}\right.$) et les molécules carbonées respectivement), l'intensité de l'émission du donneur s'exprime par la relation de Förster [7] :

$$
\begin{gathered}
\frac{I}{I_{0}}=1-\pi^{1 / 2} x \exp \left(x^{2}\right)(1-\operatorname{erf} x), \\
x=\frac{1}{2} \pi^{1 / 2} \frac{c}{c_{0}}
\end{gathered}
$$

avec

$$
\text { l'accepteur en moles/1 }
$$
et $c_{0}$ la concentration critique qui est reliée à la distance critique de transfert $R_{0}$ par $c_{0}=\frac{3000}{4 \pi N R_{0}^{3}}$, $R_{0}$ étant donné, d'après Förster, par l'expression :

$R_{0}^{6}=\frac{(9000 \log 10) K^{2}}{128 \pi^{5} n^{4} N} \eta_{0} \int_{0}^{\infty} F_{\mathrm{D}}(v) \varepsilon_{\mathrm{A}}(v) \frac{\mathrm{d} v}{v^{4}}$

Dans cette relation, $n$ désigne l'indice de réfraction de la matrice, $\eta_{0}$ le rendement quantique de l'émission du donneur, $N$ le nombre d'Avogadro et $v$ le nombre d'ondes. $K^{2}$ est un facteur d'orientation, $F_{\mathrm{D}}(v)$ la fonction normalisée à l'unité représentant la répartition spectrale énergétique de l'émission du donneur et $\varepsilon_{\mathrm{A}}(v)$ le coefficient spectral d'absorption molaire décadique de l'accepteur.

La courbe $I=f(x)$ la plus voisine de la courbe expérimentale est reproduite sur la figure 9. On en déduit $c_{0}$ et $R_{0}$, que l'on trouve égal à $11,8 \AA$ pour $\mathrm{C}_{6} \mathrm{H}_{6}$ et $13,3 \AA$ pour $\mathrm{C}_{2} \mathrm{~N}_{2}$.

Bien que la fonction (2) représente de façon satisfaisante les résultats des mesures, les valeurs de $R_{0}$ obtenues paraissent faibles pour un transfert d'énergie par interaction dipolaire.

On a cherché, en outre, à appliquer aux mélanges contenant du benzène, la relation (3) dans laquelle on a pris $\eta_{0}=1, n^{4}=6$ et $K^{2}=\frac{2}{3} . \varepsilon_{\mathrm{A}}(v)$ a été calculé à partir des données relatives au spectre d'absorption 


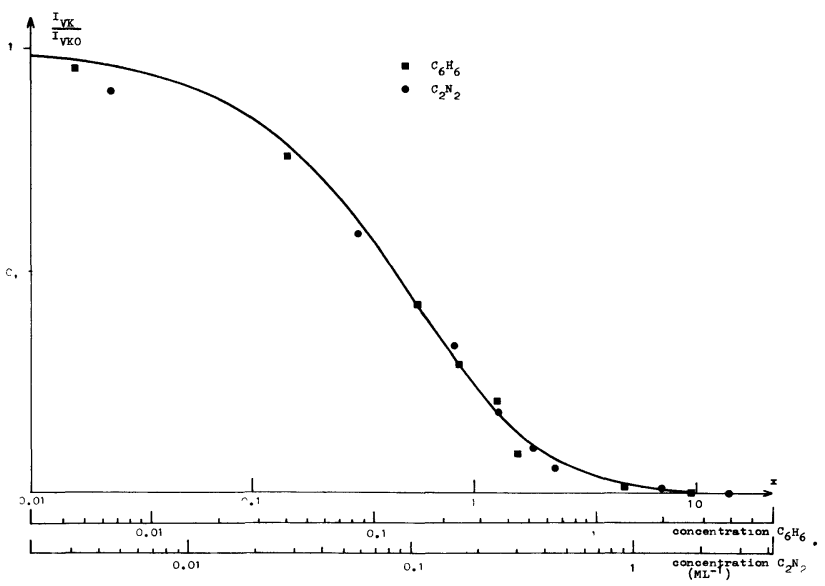

FIG. 9. - Courbe théorique obtenue d'après la relation 2 (cinétique de Förster).

$\mathrm{S} \rightarrow \mathrm{T} \mathrm{du}$ benzène publiées par Burland, Castro et Robinson [8] avec pour valeur de la force d'oscillateur $f \approx 10^{-10}$ [9]. On obtient ainsi une distance $R_{0}$ de l'ordre de $1 \AA$ pour le transfert $\mathrm{N}_{2}\left(\mathrm{~A}^{3} \Sigma_{\mathrm{u}}^{+}\right)-\mathrm{C}_{6} \mathrm{H}_{6}$. Bien que $\varepsilon_{\mathrm{A}}(v)$ soit mal connu, ce qui peut entraîner une assez forte erreur sur l'intégrale de recouvrement, il semble d'après ces résultats que les valeurs de $R_{0}$ calculées au moyen des formules (2) et (3) ne soient pas concordantes. Ainsi, l'hypothèse d'un transfert d'énergie par interaction dipôle-dipôle ne rend pas parfaitement compte de l'ensemble des résultats obtenus.

4.2.2 Transfert d'énergie par échange électronique. - En se basant sur la théorie de Dexter [10] qui s'applique aux transferts d'énergie avec changement de spin, Inokuti et Hirayama [11] ont calculé le rapport des rendements de fluorescence du donneur en présence et en l'absence d'accepteur $\eta / \eta_{0}$, que l'on peut assimiler ici au rapport des intensités $I / I_{0}$, soit :

$\frac{I}{I_{0}}=\frac{1}{\tau_{\mathrm{D}}} \int_{0}^{\infty} \exp \left[-\frac{t}{\tau_{\mathrm{D}}}-\gamma^{-3} \frac{c}{c_{0}} g\left(\frac{\exp \gamma \cdot t}{\tau_{\mathrm{D}}}\right)\right] \mathrm{d} t$,

avec $\gamma=2 R_{0} / L ; c_{0}$ et $R_{0}$ ont la même signification que dans la théorie de Förster, $L$ est le rayon moyen de Bohr pour les deux états intéressés (état excité du donneur et état fondamental de l'accepteur), $\tau_{\mathbf{D}}$ la durée de vie du donneur et $g\left(\frac{\exp \gamma \cdot t}{\tau_{\mathrm{D}}}\right)$ une fonction dont la valeur peut être calculée numériquement.

La courbe relative à $\gamma=20$ (Fig. 10), qui représente assez bien les résultats expérimentaux, conduit pour $\mathrm{C}_{6} \mathrm{H}_{6}$ et $\mathrm{C}_{2} \mathrm{~N}_{2}$ aux valeurs respectives $R_{0}=12,6 \AA$, $L=1,26 \AA$ et $R_{0}=14,5 \AA, L=1,45 \AA$. Ces distances sont du même ordre que celles trouvées par Inokuti et Hirayama pour le transfert d'énergie par échange électronique entre la benzophénone et le naphtalène.

On constate enfin que la courbe de la figure 10 est pratiquement superposable à celle que donne la

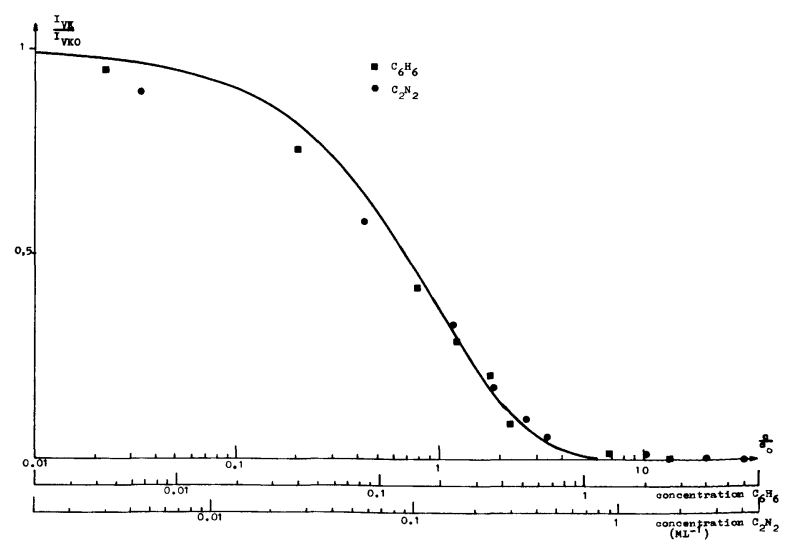

Fig. 10. - Courbe théorique déterminée à partir de la théorie de Inokuti et Hirayama.

formule de Perrin [12] :

$$
\frac{I}{I_{0}}=\exp \left(-\frac{c}{c_{0}}\right) \text {. }
$$

4.2.3 Influence de la diffusion de l'énergie. Quand l'énergie d'excitation du donneur diffuse d'une molécule à l'autre par couplage dipolaire avant d'être transférée à l'accepteur, la loi du déclin de l'émission du donneur est, d'après Yokota et Tanimoto [13] :

$$
\begin{gathered}
I(t)=I_{0} \exp \left(-\frac{t}{\tau_{\mathrm{D}}}\right) \times \\
\times \exp \left[-\frac{4}{3} \pi^{3 / 2} n_{\mathrm{A}}(\alpha t)^{1 / 2}\left(\frac{1+10,87 x+15,50 x^{2}}{1+8,743 x}\right)^{3 / 4}\right]
\end{gathered}
$$

avec $x=D \alpha^{-1 / 3} t^{2 / 3}, D$ étant le coefficient de diffusion, $\alpha$ une constante et $n_{\mathrm{A}}$ le nombre de molécules d'accepteur par unité de volume.

Le déclin n'est pas exponentiel, mais il le devient pratiquement, au bout d'un temps suffisant, et la constante de déclin de l'émission est alors donnée par :

$$
\frac{1}{\tau_{\mathrm{D}}^{\prime}}=\frac{1}{\tau_{\mathrm{D}}}+\frac{4}{3} \pi^{3 / 2}\left(\frac{15,50}{8,743}\right)^{3 / 4} \mathrm{D}^{3 / 4} \alpha^{1 / 4} n_{\mathrm{A}}
$$

En ne tenant pas compte de l'écart avec la loi exponentielle pendant la première phase du déclin, on obtient pour le rapport des rendements quantiques du donneur en présence et en l'absence de l'accepteur, et par suite pour le rapport des intensités :

$$
\frac{I}{I_{0}}=\frac{\tau_{\mathrm{D}}^{\prime}}{\tau_{\mathrm{D}}}=\frac{1}{1+k c}
$$

où $c$ désigne comme dans les formules précédentes, la concentration du composé $X$. La relation (6), qui est identique à la formule de Stern et Volmer [14], ne représente pas correctement les résultats expérimentaux, ainsi qu'on le voit sur la figure 11.

Comme, d'autre part, la constante de diffusion $D$ est proportionnelle à la concentration du donneur [15], le coefficient $k$ devrait croître en même temps que la 


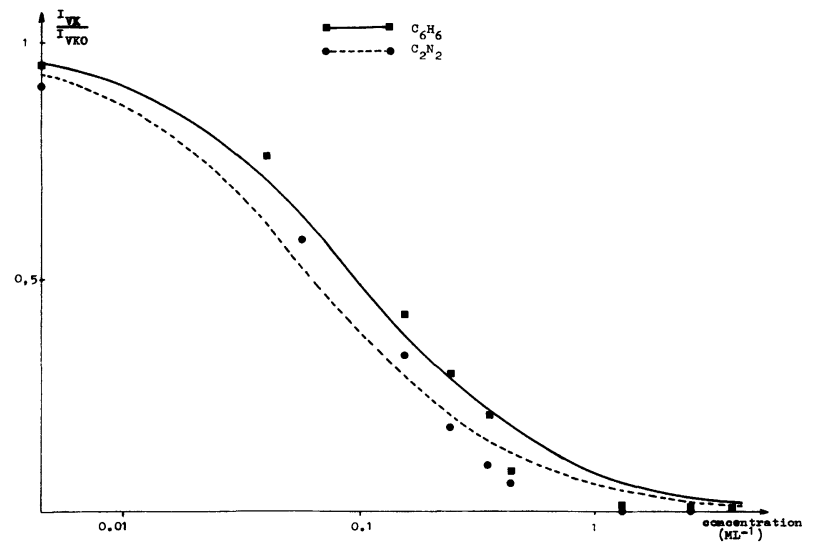

Fig. 11. - En trait plein et en pointillé, courbes obtenues d'après la relation de Stern-Volmer pour le benzène et pour le cyanogène.

concentration de l'azote dans le mélange utilisé, ce qui n'est pas conforme à l'expérience, puisque les courbes $I_{\mathrm{VK}} / I_{\mathrm{VKo}}=f(c)$ obtenues pour les deux concentrations d'azote 1 et $6 \%$ sont pratiquement superposées (Fig. 12).

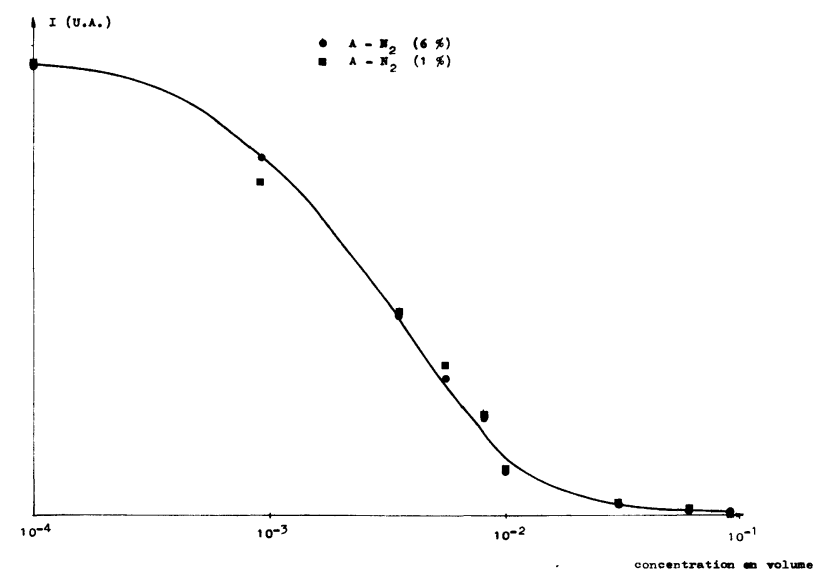

Fig. 12. - Comparaison des valeurs expérimentales de l'intensité de la bande (0-7) de Vegard-Kaplan pour des mélanges contenant respectivement $1 \%$ et $6 \%$ d'azote.

Il semble donc que la diffusion de l'énergie d'excitation par interaction dipolaire entre les molécules d'azote n'ait pas une grande influence. Comme d'autre part la distance entre ces molécules est de l'ordre de $20 \AA$, lorsque la concentration est égale à $1 \%$, l'interaction par échange électronique doit être relativement faible. Aussi a-t-on admis dans le modèle proposé que le transfert d'énergie entre les molécules du donneur ne jouait pas un rôle important.

4.2.4 Effet de la diffusion moléculaire. - L'aspect discontinu de la courbe de variation thermique de l'intensité des bandes de Vegard-Kaplan et l'évolution avec la température des intensités respectives des bandes de l'azote et du produit carboné conduisent à penser que la diffusion moléculaire peut intervenir. La présence de nombreuses imperfections dans les matrices obtenues par condensation rapide d'une vapeur et l'énergie importante libérée par recombinaison atomique doivent, en effet, favoriser la diffusion des molécules $\mathrm{N}_{2}\left(\mathrm{~A}^{3} \Sigma_{\mathrm{u}}^{+}\right)$formées.

Puisque les formules (4) et (5) donnent des résultats pratiquement identiques, on a considéré, comme l'avaient fait Frank et Vavilov [16], que la relation de Perrin est applicable. Cela suppose toutefois que la vitesse de diffusion soit faible, puisque la théorie d'Inokuti et Hirayama implique que les transferts d'excitation n'aient lieu qu'entre des donneurs et des accepteurs dont la distance demeure sensiblement constante.

Lorsqu'on ne tient pas compte de la diffusion, on peut écrire que la probabilité d'émission d'une bande de Vegard-Kaplan pendant le temps $\mathrm{d} t$ est proportionnelle à : $\exp \left(-c / c_{0}\right) \exp \left(-t / \tau_{\mathrm{D}}\right) \mathrm{d} t$, où $\tau_{\mathrm{D}}$ est la durée de vie des molécules $\mathrm{N}_{2}\left(\mathrm{~A}^{3} \Sigma_{\mathrm{u}}^{+}\right)$. En désignant par $t_{\mathrm{m}}$ l'intervalle de temps moyen entre la formation d'une molécule d'azote métastable et sa rencontre avec une molécule $X$ par diffusion, ce qui entraîne sa désactivation, la probabilité d'émission devient :

$$
\exp \left(-\frac{c}{c_{0}}-\frac{t}{\tau_{\mathrm{D}}}-\frac{t}{t_{\mathrm{m}}}\right) \mathrm{d} t
$$

D'après la théorie d'Einstein-Smoluchovski [17], le flux de diffusion à travers la sphère de rayon $R_{0}$ a pour valeur :

$$
n=\frac{4 \pi N R_{0} D}{1000} c\left[1+R_{0}(\pi D t)^{-1 / 2}\right],
$$

où $D$ est la somme des constantes de diffusion du donneur et de l'accepteur.

On obtient une valeur approchée du deuxième terme de $n$ en remplaçant $t$ par $\tau_{\mathrm{D}}$ et, en écrivant que $t_{\mathrm{m}}$ est égal à $1 / n$, il vient par intégration :

$$
\begin{aligned}
I_{\mathrm{VK}} & =I_{\mathrm{VKO}} \frac{\exp \left(-c / c_{0}\right)}{1+\frac{4 \pi N R_{0} D \tau_{\mathrm{D}}}{1000}\left[1+R_{0}\left(\pi D \tau_{\mathrm{D}}\right)^{-1 / 2}\right] c} \\
& =I_{\mathrm{VKO}} \frac{\exp \left(-c / c_{0}\right)}{1+k c}
\end{aligned}
$$

La figure 13 montre que la relation (7) représente mieux les résultats expérimentaux que les formules (5) et (6). On trouve pour le benzène $c_{0}=0,516 \mathrm{ML}^{-1}$ et $k=5,16$, d'où le rayon d'interaction $R_{0}=9,2 \AA$, et pour le cyanogène $c_{0}=0,415 \mathrm{ML}^{-1}, k=7,6$ et $R_{0}=9,8 \AA$. En prenant pour $\tau_{\mathrm{D}}$ la durée de vie de la molécule $\mathrm{N}_{2}\left(\mathrm{~A}^{3} \Sigma_{\mathrm{u}}^{+}\right)$dans l'argon solide, en l'absence de produit étranger, on en déduit les constantes de diffusion :

$D=1,2 \times 10^{-14} \mathrm{~cm}^{2} \mathrm{~s}^{-1}$, dans le cas du benzène, $D=1,9 \times 10^{-14} \mathrm{~cm}^{2} \mathrm{~s}^{-1}$, dans celui du cyanogène.

Les longueurs de diffusion $\sqrt{2 D \tau_{\mathrm{D}}}$ vaudraient respectivement $9 \AA$ et $11,5 \AA$, c'est-à-dire qu'elles seraient 


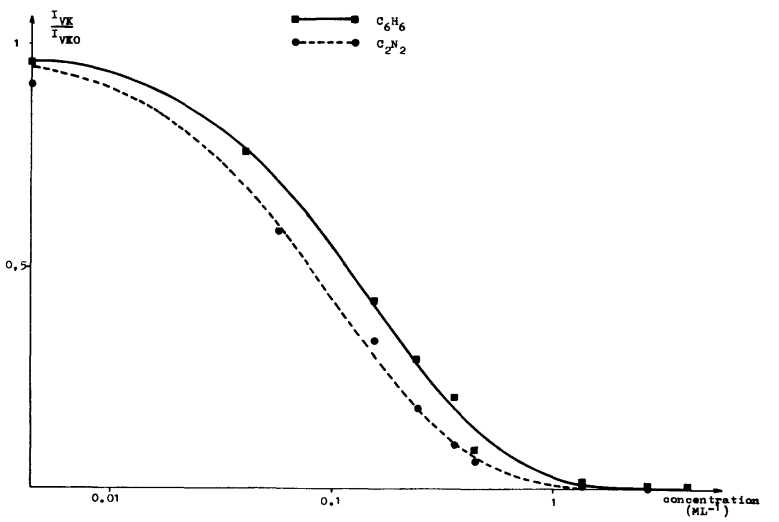

FIG. 13. - En trait plein et en pointillé, courbes obtenues d'après la formule (7) pour le benzène et pour le cyanogène.

du même ordre que $R_{0}$. En fait, la valeur de $\tau_{\mathrm{D}}$ est abaissée par suite du transfert d'énergie et pour la concentration relative au maximum de l'émission du composé carboné, on trouve des longueurs de diffusion égales respectivement à 3,5 et $3,7 \AA$ pour les deux composés. Ainsi, les conditions d'application de la théorie du transfert d'énergie par échange électronique ne sont qu'approximativement réalisées. De ce fait et en raison des simplifications introduites dans le calcul effectué, les valeurs obtenues donnent seulement un ordre de grandeur des différents paramètres.

4.3 EXTINCTION DE L'ÉMISSION DU COMPOSÉ POLYATOMIQUE PAR AUGMENTATION DE SA CONCENTRATION. - On a cherché à interpréter au moyen des hypothèses précédentes, les courbes des figures 2 et 3 qui représentent les variations avec la concentration $c$ de l'une des bandes du benzène et du cyanogène.

En désignant respectivement par $n_{0}$ et par $n$ les nombres des molécules $\mathrm{N}_{2}\left(\mathrm{~A}^{3} \Sigma_{\mathrm{u}}^{+}\right)$qui rayonnent en l'absence et pour une concentration $c$ du produit carboné, on peut écrire $: \frac{n}{n_{0}}=\frac{I_{\mathrm{VK}}}{I_{\mathrm{VKo}}}$. Si l'on admet que toutes les transitions sans émission correspondent à un transfert d'énergie aux molécules polyatomiques $X$, le nombre de molécules $X$ excitées est, par molécule d'azote métastable créée :

$$
\frac{n_{0}-n}{n_{0}}=1-\frac{\exp \left(-c / c_{0}\right)}{1+k c} \text {. }
$$

Cette hypothèse constitue une approximation justifiée par le fait que la raie $N\left({ }^{2} \mathrm{D}-{ }^{4} \mathrm{~S}\right)$ est peu intense, même en l'absence du composé $\mathrm{X}$, et que la concentration des molécules $\mathrm{N}_{2}\left(\mathrm{~A}^{3} \Sigma_{\mathrm{u}}^{+}\right)$est très inférieure à celle de $\mathrm{X}$ dans la matrice.

On sait que dans les solutions contenant des teneurs élevées de composés organiques, il peut se former des dimères et que, d'après de nombreux auteurs, il en résulte une extinction de l'émission de ce composé. On admettra qu'un mécanisme analogue est responsable de l'extinction de la luminescence observée ici.
Si l'on désigne par $\tau_{\mathrm{A}}$ la durée de vie de la molécule excitée $\mathrm{X}(\mathrm{T})$ et par $t_{\mathrm{m}}^{\prime}$ l'intervalle de temps moyen entre l'excitation de $\mathrm{X}$ et l'entrée d'une autre molécule $X$ dans le volume actif de $X(T)$, on peut écrire que la probabilité d'émission du système $T \rightarrow S$ pendant l'intervalle de temps $\mathrm{d} t$, est de la forme :

$$
\exp \left(-\frac{c}{c_{0}^{\prime}}-\frac{t}{\tau_{\mathrm{A}}}-\frac{t}{t_{\mathrm{m}}^{\prime}}\right) \mathrm{d} t
$$

Le nombre de molécules $\mathrm{X}(\mathrm{T})$ qui émettent est alors en régime stationnaire, proportionnel à :

$$
\left[1-\frac{\exp \left(-c / c_{0}\right)}{1+k c}\right] \int_{0}^{\infty} \exp \left(-\frac{c}{c_{0}^{\prime}}-\frac{t}{\tau_{\mathrm{A}}}-\frac{t}{t_{\mathrm{m}}^{\prime}}\right) \mathrm{d} t
$$

D'où l'intensité $I_{\mathbf{X}}$ de la bande considérée :

$$
I_{\mathrm{X}}=K\left[1-\frac{\exp \left(-c / c_{0}\right)}{1+k c}\right] \frac{\exp \left(-c / c_{0}^{\prime}\right)}{1+k^{\prime} c}
$$

avec

et

$$
c_{0}^{\prime}=\frac{3000}{4 \pi R_{0}^{\prime 3} N}
$$

$$
k^{\prime}=\frac{4 \pi N R_{0}^{\prime} D^{\prime} \tau_{\mathrm{A}}}{1000}\left[1+R_{0}^{\prime}\left(\pi D^{\prime} \tau_{\mathrm{A}}\right)^{-1 / 2}\right]
$$

Les valeurs des constantes $c_{0}^{\prime}, R_{0}^{\prime}$ et $D^{\prime}$ obtenues dans le cas du benzène sont respectivement :

$c_{0}^{\prime}=6,3 \mathrm{ML}^{-1}, R_{0}^{\prime}=4 \AA, D^{\prime}=0,7 \times 10^{-16} \mathrm{~cm}^{2} \mathrm{~s}^{-1}$

On constate sur la figure 14 que la formule (9) représente assez bien les résultats expérimentaux, sauf aux très faibles concentrations, ce qui pourrait s'expliquer par le fait que la concentration est alors déterminée avec une assez forte incertitude.

Le calcul précédent ne peut fournir qu'un ordre de grandeur de $R_{0}^{\prime}$, mais puisqu'il est très petit, on en conclut que le résultat obtenu n'est pas en désaccord avec l'hypothèse de la formation de dimères.

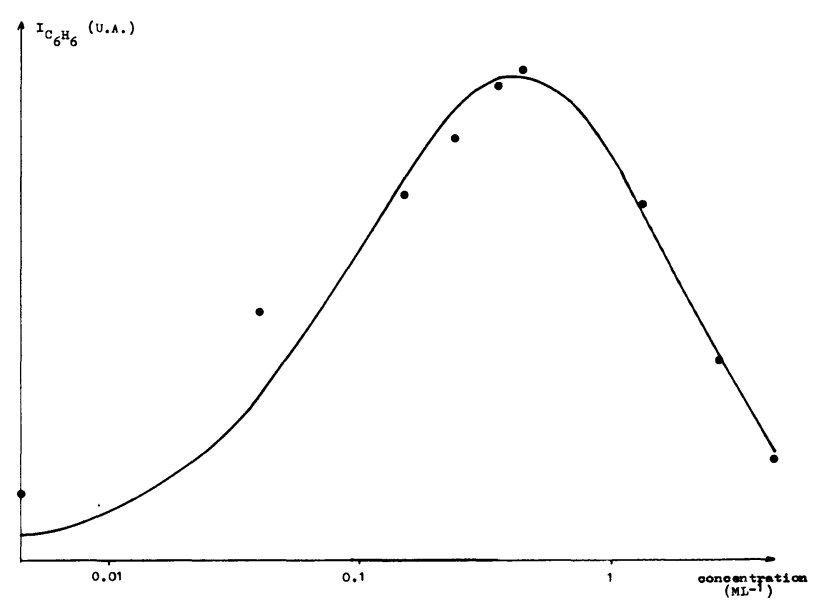

Fig. 14. - Courbe obtenue d'après la relation (9) pour le benzène. 
De plus, le coefficient de diffusion du benzène étant extrêmement faible, cela signifie que cette molécule diffuse très peu. Il en résulte que la valeur de $D$, trouvée dans l'étude de l'interaction $\mathrm{N}_{2}\left(\mathrm{~A}^{3} \Sigma_{\mathrm{u}}^{+}\right)-\mathrm{C}_{6} \mathrm{H}_{6}$ (8), se rapporte aux molécules d'azote métastables. On constate qu'elle est de l'ordre de grandeur de celle qu'ont obtenue récemment Meyer et Metzger [18] pour l'oxygène dans une matrice d'argon à $33 \mathrm{~K}$, soit $1,2 \times 10^{-12 \pm 2} \mathrm{~cm}^{2} \mathrm{~s}^{-1}$.

Pour le cyanogène, on trouve un volume actif pratiquement nul, les valeurs numériques obtenues étant sans signification. La figure 15 permet de comparer aux points expérimentaux la courbe représentant la fonction (9) avec $1 / c_{0}^{\prime}=0$ et $k^{\prime}=1,26$. On voit que, comme dans le cas du benzène, l'accord est seulement approché. En utilisant la relation (8) pour calculer la constante de diffusion du cyanogène et prenant $\tau_{\mathrm{A}}$ égal à $1 \mathrm{~s}$ [2], on déduit de (9) le rayon d'action $R_{0}^{\prime}=2 \AA$. Il serait donc très petit, ce qui explique qu'on n'ait pu déterminer directement le volume actif à partir des données expérimentales.

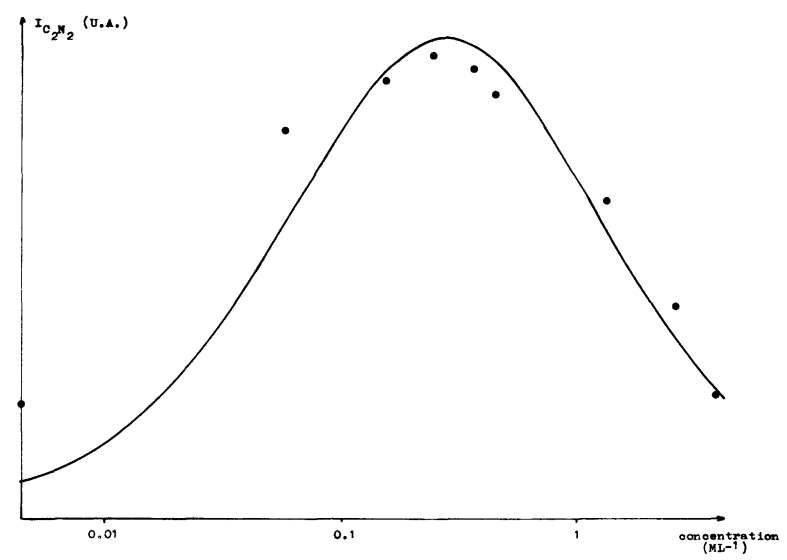

FIG. 15. - Courbe obtenue d'après la relation (9) pour le cyanogène.

5. Conclusion. - Les résultats obtenus dans cette étude peuvent s'interpréter en considérant que l'exci- tation des molécules carbonées par l'azote actif dans des matrices azote-argon à basse température est due, ainsi qu'on l'avait supposé antérieurement, à un transfert d'énergie entre les molécules $\mathrm{N}_{2}\left(\mathrm{~A}^{3} \Sigma_{\mathrm{u}}^{+}\right)$ produites par recombinaison d'atomes $\mathrm{N}\left({ }^{4} \mathrm{~S}\right)$ et les molécules polyatomiques. Bien que les phénomènes soient complexes, on a cherché au moyen d'hypothèses simplificatrices à déterminer l'ordre de grandeur des distances d'interaction. Les valeurs trouvées — une dizaine d'angströms - semblent indiquer qu'il s'agit d'un transfert d'énergie par échange électronique, sans qu'une conclusion définitive puisse être donnée pour l'instant.

Lorsqu'on opère en condensant les gaz en régime stationnaire sur une paroi refroidie par l'hydrogène liquide, la couche émissive est la couche superficielle du dépôt, dont la température est probablement supérieure à celle de la paroi (voisine de $25 \mathrm{~K}$ dans nos expériences). Etant donné qu'une matrice d'azote ne peut être considérée comme parfaitement rigide audessus de $17 \mathrm{~K}$ et qu'il en est de même pour une matrice d'argon à partir de $20 \mathrm{~K}$ [19], et puisque, d'autre part, la recombinaison des atomes d'azote est fortement exothermique, il paraît assez vraisemblable que dans les matrices mixtes utilisées ici, une certaine diffusion des molécules insérées dans la partie superficielle de la couche puisse se produire. C'est pourquoi dans le mécanisme proposé qui rend assez bien compte des résultats des mesures, on a admis l'existence d'une telle diffusion. Celle-ci serait très faible pour les molécules de benzène, tandis qu'elle serait plus importante pour les molécules d'azote excitées et peut-être aussi pour celles de cyanogène.

Enfin, les très faibles distances d'interaction que l'on a trouvées dans l'étude de l'extinction par concentration de la luminescence des produits carbonés sont compatibles avec l'attribution de ce phénomène à la présence de dimères aux concentrations élevées, comme c'est souvent le cas pour les solutions de composés organiques.

\section{Bibliographie}

[1] Faure, E., Valadier, F., Bergeon, R., Janin, J., C. R. Hebd. Séan. Acad. Sci. 263 (1966) 1234; J. Physique Collq. 28 (1967) C3-143; C. R. Hebd. Séan. Acad. Sci. 265 (1967) 1432.

Faure, E., Valadier, F., Janin, J., C. R. Hebd. Séan. Acad. Sci. 269 (1969) 431; C. R. Hebd. Séan. Acad. Sci. 271 (1970) 419; C. R. Hebd. Séan. Acad. Sci. 272 (1971) 532.

[2] Meyer, J. A., Stedman, D. H., SetSer, D. W., J. Mol. Spectrosc. 44 (1972) 206.

[3] Peyron, M., Broida, H. P., J. Chem. Phys. 30 (1959) 139; 32 (1960) 1068.

Peyron, M., Hörl, E. M., Brown, H. W., Broida, H. P., J. Chem. Phys. 30 (1959) 1304.

HöRL, E. M., J. Mol. Spectrosc. 3 (1959) 425.

SChOEN, L. J., RebBeRT, R. E., J. Mol. Spectrosc. 3 (1959) 417.

[4] Dressler, K., Mémoires Soc. Roy. Sci. Liège 20 (1970) 357.

[5] Tinti, D. S., Robinson, G. W., J. Chem. Phys. 49 (1968) 3229.

[6] Fontana, B. J., J. Chem. Phys. 29 (1958) 1668; J. Chem. Phys. 31 (1959) 148.

[7] Förster, Th., Naturwiss. 33 (1946) 166; Ann. Physik 2 (1948)
55; Z. Naturforsch. 4a (1949) 321; Disc. Faraday Soc. 27 (1959) 7.

[8] Burland, D. M., Castro, G., Robinson, G. W., J. Chem. Phys. 52 (1970) 4100.

[9] Wright, M. R., Frosch, R. P., Robinson, G. W., J. Chem. Phys. 33 (1960) 934.

Robinson, G. W., J. Mol. Spectrosc. 6 (1961) 58.

Colsoin, S. D., Bernstein, Ë. R., J. Chem. Phys. 43 (1956) 2661.

[10] Dexter, D. L., J. Chem. Phys. 21 (1953) 836.

[11] Inokuti, M., Hirayama, F., J. Chem. Phys. 43 (1965) 1978.

[12] Perrin, F., C. R. Hebd. Séan. Acad. Sci. 178 (1924) 1978.

[13] Yoкота, M., Tanimoto, O., J. Phys. Soc. Japan 22 (1967) 779.

[14] Stern, O., Volmer, M., Physik. Z. 20 (1919) 183.

[15] Weber, M. J., Phys. Rev. B 4 (1971) 2932.

[16] Frank, J. M., Vavilov, S. I., Z. Physik 69 (1931) 100.

[17] Von Smoluchowski, M., Z. Phys. Chem. 92 (1917) 129.

[18] Meyer, B., Metzger, J. L., J. Chem. Phys. 60 (1974) 796.

[19] Smith, G., Henry, S., Blount, C. E., J. Mol. Spectrosc. 35 (1970) 61. 